Research Article

\title{
An Experiment-Based Simplified Method for the Model of Building Groups in CFD Simulation
}

\author{
Hongling Guo $\mathbb{D}^{1},{ }^{1}$ Ying Zhou $\mathbb{D}^{1},{ }^{1}$ Yingxin $\mathrm{Li},{ }^{1}$ Zhihui Zhang, ${ }^{1}$ and Heng $\mathrm{Li}^{2}$ \\ ${ }^{1}$ Department of Construction Management, Tsinghua University, Haidian District, Beijing 100084, China \\ ${ }^{2}$ Department of Building and Real Estate, The Hong Kong Polytechnic University, Hung Hom, Hong Kong, China \\ Correspondence should be addressed to Hongling Guo; hlguo@tsinghua.edu.cn
}

Received 1 August 2020; Revised 9 December 2020; Accepted 28 March 2021; Published 14 April 2021

Academic Editor: Luigi Aldieri

Copyright (c) 2021 Hongling Guo et al. This is an open access article distributed under the Creative Commons Attribution License, which permits unrestricted use, distribution, and reproduction in any medium, provided the original work is properly cited.

Computational Fluid Dynamics (CFD) has been widely used in the simulation and analysis of community or urban wind environments. However, the CFD-based wind simulation of large-scale building groups usually consumes a lot of computing resources with high computing costs. To improve the efficiency of CFD-based wind simulation, this paper presents an experimentbased simplified method for the model of building groups. Two rectangular buildings are adopted as the basic unit and four control parameters $(B / L, W / L, H / L$, and $D / L)$ are selected as the experiment factors to analyze the geometrical relationship of the two buildings. Note that $L, W$, and $H$, respectively, represent the windward edge length, width, and height of a building, $B$ is building interval distance, and $D$ is the distance between two building center axes. Then, a single factor experiment and an orthogonal experiment are designed and performed to determine the reasonable value range of each factor. Based on the experiment results and actual situation, the value ranges of four factors for the simplification of building group models are determined as follows: $B / L \in\{0,1.5\}, W / L \in\{0,2\}, D / L \in\{0,0.25\}$, and $H / L \in\{0,1\}$. Furthermore, a real case is presented to evaluate the performance of the proposed simplified method. The results indicate that the simplified method is able to improve the efficiency of CFD-based wind simulation of building groups, with the number of buildings decreasing from 620 to 395 (by $36.3 \%$ ), and the number of tetrahedral grids decreasing from 8,832,199 to 7,766,778 (by 12.1\%). Thus, this research contributes to the CFD-based wind simulation method of large-scale building groups and the analysis of the urban wind environment.

\section{Introduction}

Urban wind environment has attracted growing attention from urban planners. Previous research has shown that outdoor temperature is increased by $1.9^{\circ} \mathrm{C}$ with the wind speed decreasing from $1 \mathrm{~m} / \mathrm{s}$ to $0.3 \mathrm{~m} / \mathrm{s}$, and the wind speed for thermal comfort in subtropical cities in summer is about $1.6 \mathrm{~m} / \mathrm{s}$ [1]. Besides, the planning of urban wind environment has a significant impact on urban climate issues such as urban heat island effect, pollutant diffusion, usage of wind energy, and air quality [2-4]. Good wind environment contributes to a pleasant human living environment and plays an important role in constructing green ecological cities.

Simulation methods and technologies are being used in the analysis of urban wind environment. Particularly,
Computational Fluid Dynamics- (CFD-) based simulation methods are attracting urban planners. Early CFD was developed for spacecraft design under special conditions. In the late 1970s, NASA designed experimental aircraft Highly Operational Aircraft Technology (HIMAT) [5] with CFD technology. With the improvement of computational performance, the application of CFD has been expanded to different industries, such as automobile and engine design, shipbuilding, civil engineering, and environmental engineering. In the study on urban wind environment, compared with traditional research methods (e.g., field measurement and wind tunnel test), CFD is more cost-effective, timesaving, and information-detailed. It can also simulate different spatial dynamics like hurricanes. Otherwise, CFD simulation can be applied to promote the accurate recognition of urban wind environment in the urban planning 
stage, thus improving the quality of architectural design and urban planning.

However, in the CFD-based wind simulation of largescale building groups, millions of grids need to be generated to describe the flow field exactly and completely, which requires high computational performance. In addition, CFD needs to rebuild the model of building groups and thus slow down relevant simulation as design alterations take place in urban planning. Therefore, it is obvious that the reasonable simplification of geometric models of building groups can enhance the efficiency of CFD-based wind simulation. To solve the aforementioned problem, this paper proposes a simplified method for the model of a cluster of buildings to reduce the computational cost from the perspective of a geometric model by the reasonable consolidation (simplification) of building models. It also considers the balance between computational efficiency and accuracy to promote the application of CFD in urban wind environment simulation.

\section{Literature Review}

The research on the CFD-based simulation of urban wind environment can be generally classified into two categories, i.e., the wind simulation of a single building and that of building groups. The former is mainly about the analysis of airflow inside or around a single building, focusing on the relationship between the building structure and wind environment. The wind environment mainly refers to indoor and outdoor natural ventilation and airflow. According to different types of structures, there are atrium buildings [6], sunroof buildings [7], serrated roof buildings [8], and other structures [9-12]. Reasonable building exterior design and elevation layout can facilitate air exchange, improve thermal comfort, and reduce building energy consumption. Thus, the research on the wind environment of a single building becomes more and more popular in the design of green buildings.

Moreover, the CFD-based wind simulation of building groups focuses on airflow among city streets, public places, and different buildings. According to the scale, building groups in an urban area can be classified into urban (up to 10 or $20 \mathrm{~km}$ ), neighborhood (up to 1 or $2 \mathrm{~km}$ ), and street scale (less than 100-200 m), to which different simulation accuracy and modeling methods are applicable [1]. In the street scale, Omar et al. analyzed the outdoor ventilation of different buildings combination structures in different wind directions and concluded that the central space formed by adjacent buildings and the building facing main wind are conducive to airflow [13]. Wang et al. used CFD to study the relationship between wind and orthogonal building layout and found that orthogonal building layout has higher wind energy density than a single building [2]. James explored the effects of building disturbances on natural ventilation by comparing airflow efficiency under conventional and misaligned rows; the results indicate that reasonable alternate distribution and building orientation can improve ventilation efficiency [14].

As for the neighborhood scale, previous research shows that the layout of streets has a great impact on the wind environment. Cao et al. analyzed the relationship between street direction and wind speed to show the impacts on urban thermal environment, which is significant to the street design [15]. Ramponi et al. [16] did the CFD simulation of wind environment in equal width or unequal width parallel streets in different areas and concluded that ventilation reduces in the downwind districts [3]. In addition, some researchers studied the wind environment of building groups with different properties. Huang et al. used 2D CFD to analyze the impact of gable roofs on airflow and pollutant diffusion in urban street canyons [3]. Hang compared the natural ventilation of different wind directions in open and semiopen urban spaces [17]. Yuan et al. developed a model with CFD to simulate air pollution dispersion and natural ventilation in high-density areas and found that reasonable urban planning can improve the air quality indoor and in street canyons [18]. Furthermore, Yuan et al. applied the semiempirical model to simulate the wind environment of high-density urban areas and developed a modeling-mapping approach to balancing modeling cost with result accuracy, which is statistically significant to evaluating wind speed in large-scale districts but lacks the theoretical support of physics [18].

At the urban scale, there is little research, particularly on the simplified method of building models. Tominaga et al. specified the boundary condition in the CFD simulation of a single building by substituting some simplified geometries for a cluster of buildings [19]. Other relevant research did not propose a quantifiable method to simplify the model of building groups. In order to simulate the urban wind environment, $\mathrm{Wu}$ et al. built a simplified model, in which the ratio of building space to building height and the ratio of separation distance to building length were selected as key parameters for the analysis of environmental effects [20]. However, the comparative analysis was not quantified. Moreover, the model is more ideal and thus limited to a certain of buildings because the selection and analysis of parameters are not comprehensive. For instance, the axis offset of staggered layout buildings is neglected and the influence of some parameters on the simplification of building models not analyzed.

Based on the current research, this research will develop a simplified method for the model of building groups with comprehensive parameters by simulating and analyzing the relationship between buildings and the mechanism of the wind environment without affecting the general trend of regional airflow.

\section{Methodology}

3.1. Definition of Model Simplification. It is difficult to comprehensively analyze the impact of building layout on wind environment as the layout of building groups varies from one to another. Previous research found that the differences in building layout [21] and elevation [22] had obvious effects on the wind environment. Therefore, two identical rectangular buildings in parallel are extracted as the ideal basic unit before the simplification to represent the layout of adjacent buildings in most building groups. As 
shown in Figure 1, the simplification in this research means that the basic unit can be replaced with a parallelogram formed by the contour line of the building group. European Cooperation in the Field of Science and Technical Research (COST) suggests that an initial choice should be at least 10 cells per building side and 10 cells per cube root of building volume in CFD simulation [23]. Under the resolution requirements of COST, the simplification of the model will theoretically reduce the number of grids with consuming less time and labor. Besides, the reduction of strange-shaped space can also improve grid quality and save computational cost.

3.2. Selection of Control Parameters. The simplification of building groups needs to comply with certain rules. Control parameters are used to determine whether or not the model of a building group can be combined or simplified. Control parameters are specific quantitative expressions of complicated model space relationships and can reflect the characteristics of the complex shape to a certain extent. Some researchers used the ratio of building spacing and offset distance to the size of building to express the characteristics of building groups, while others used the ratio of windward area and projected area to building area as control parameters. The spatial relationship of a group of buildings is also selected as the control parameters of the simplified method in this research, but neglecting those factors difficult to quantify, such as complex topography, water, and vegetation. To determine the control parameters, we propose a basic building unit consisting of two identical rectangular buildings arranged along the wind direction, involving five variables, i.e., the dimensions of a building model (windward edge length $(L)$, width $(W)$, and height $(H)$ ) and the building group offsets (building interval distance $(B)$ and distance between two building center axes $(D)$ ) (see Figure 2). To make control parameters dimensionless and universal, we used the ratios of different variables as the control parameters. Considering that the windward edge length $(L)$ has a great impact on the range of the wind shadow area behind a building, in order to reduce the system error caused by wind shadow, the ratios of $W, H, B$, and $D$ to $L$ (i.e., $B / L, W / L, H / L$, and $D / L)$ are selected as the control parameters for subsequent experiments.

3.3. Determination of Value Ranges of the Control Parameters. Single factor experiment and orthogonal experiment are performed in this research to determine the appropriate value range of each control parameter for the model simplification of building groups. Figure 3 illustrates the entire process of experiments from a technical perspective. Note that $[\alpha, \beta]$ and $\left[\alpha^{\prime}, \beta^{\prime}\right]$ are to, respectively, represent the results (i.e., the value ranges of control parameters) of single factor experiment and orthogonal experiment, and $\mathrm{P}$ represents the selected parameters (i.e., $B / L, W / L, H / L$, and $D /$ $L$ ), thus meaning $\alpha \leq \alpha^{\prime} \leq P \leq \beta^{\prime} \leq \beta$. Only one factor is changing in the single factor experiment, with other factors remaining fixed to analyze the specific effect of the changing factor on experimental results. Besides, as the preparation of
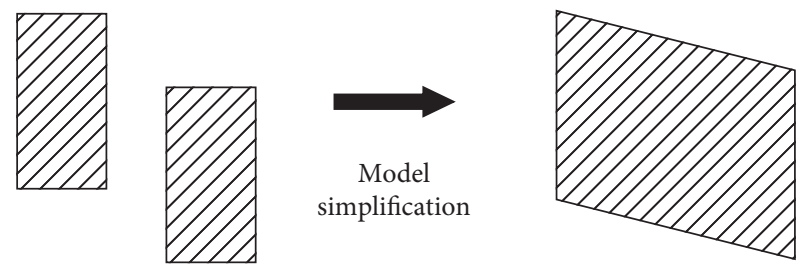

FIgURE 1: Schematic diagram of model simplification (plan view).

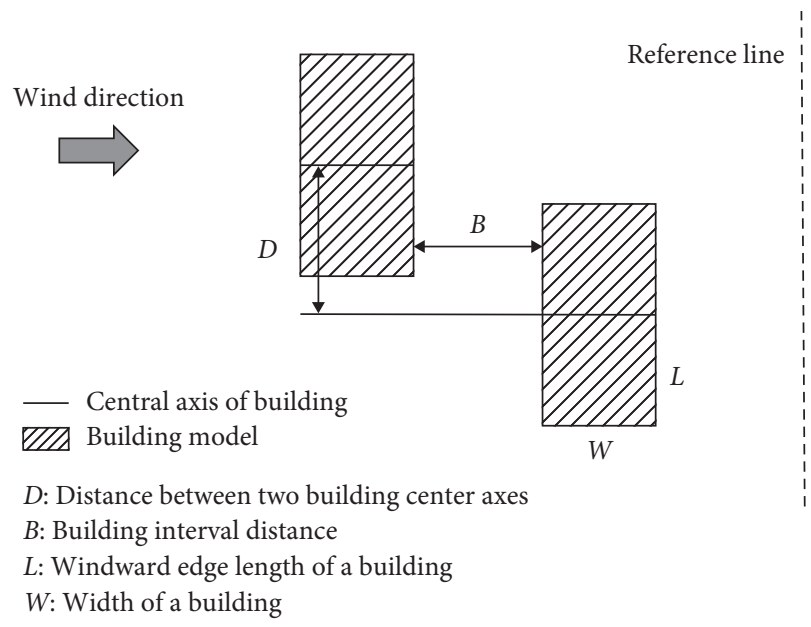

Figure 2: Variables in the basic unit (building group).

the orthogonal experiment, the single factor experiment explores the function of each parameter to figure out the appropriate value range of each control parameter in the design process of the orthogonal experiment.

Orthogonal experiment using orthogonal tables for multifactor analysis can reliably infer the conclusion of a comprehensive experiment through a small number of selected representative tests with an optimized experimental scheme. Based on the results of the single factor experiment, the orthogonal experiment can not only analyze the interaction between different factors but also significantly reduce test times. And the effects of different control parameters on experimental results are ordered by intuitive analysis and variance analysis to select the optimal value and the suboptimal value as the optimal value range of each control parameter. Finally, the coordinated control indexes are verified by suitability verification.

\section{Experiment Setup}

4.1. Design of Single Factor Experiment. Four groups of single factor experiment were designed to analyze the four factors $(B / L, W / L, H / L$, and $D / L)$. Considering the actual size of general buildings, $L$ was set to $30 \mathrm{~m}$. Besides, the control parameters of the initial model in the single factor experiment were set as follows: $B / L=0.5, W / L=1, H / L=1$, and $D / L=0$. Figure 4 shows the initial model for the single experiment, in which $L * W * H=30 \mathrm{~m} * 30 \mathrm{~m} * 30 \mathrm{~m}, \quad B=15 \mathrm{~m}$, and $D=0 \mathrm{~m}$. In the same way, the upper limit values of $B / L, W / L$, and $H / L$ were all set to 3 , with six levels in equidistant distribution. As the projected area of the upwind building to the 


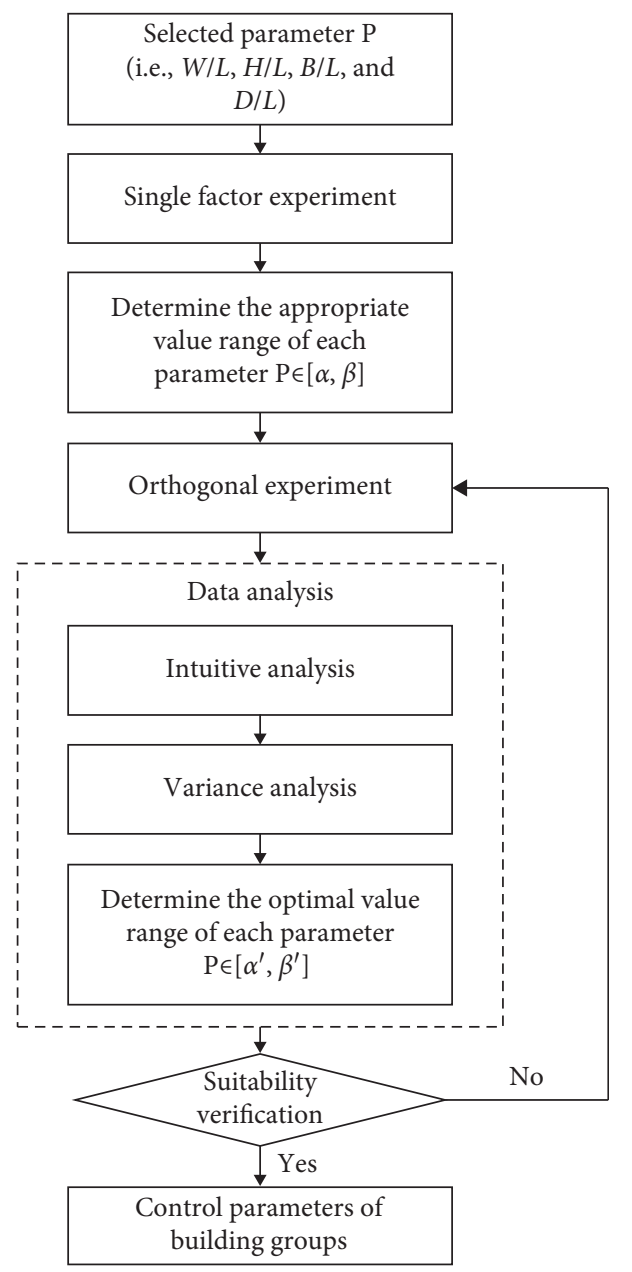

Figure 3: Technical roadmap of the entire experiment.

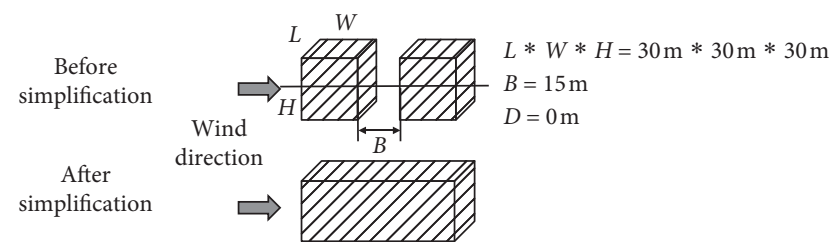

Figure 4: The initial model of the single factor experiment.

downwind building is greater than or equal to 0 , which meant $D \leq L$, the upper limit of $D / L$ was 1 and set to six levels with the equidistant distribution. The corresponding control parameters of the single factor experiment are shown in Table 1, while other control parameters stay the same as those of the initial model.

4.2. Design of Orthogonal Experiment. The value ranges of four factors obtained from the single factor experiment were set to 4 levels in the following orthogonal experiment. According to the selection principle of the orthogonal table (i.e., to select a smaller experiment table based on the number of factors less than that of the column in an
TABLE 1: The level of control parameters of the single factor experiment.

\begin{tabular}{lcccc}
\hline Level & $B / L$ & $W / L$ & $H / L$ & $D / L$ \\
\hline 1 & 0.5 & 0.5 & 0.5 & 0 \\
2 & 1 & 1 & 1 & 0.2 \\
3 & 1.5 & 1.5 & 1.5 & 0.4 \\
4 & 2 & 2 & 2 & 0.6 \\
5 & 2.5 & 2.5 & 2.5 & 0.8 \\
6 & 3 & 3 & 3 & 1 \\
\hline
\end{tabular}

orthogonal table and the number of factor levels same as that of the orthogonal table), for the 4-factor-4-level experiment, a 5-factor-4-level orthogonal experiment table L16 $\left(4^{5}\right)$ was selected (see Table 2). In comparison with the number of comprehensive tests, that of the test was effectively decreased from 256 (44) to 16.

4.3. Result of the Calculation Method. In this research, deviation, as the criterion to evaluate experiments, refers to the speed changes at the reference line after simplification and consolidation.

4.3.1. Selection of Reference Line. As the basis of the deviation analysis, the reference line was selected as follows:

(1) The line located at $20 \mathrm{~m}$ behind the building group is in a general distance behind the model to show the influence of the front buildings on the rear buildings

(2) The line is $1.5 \mathrm{~m}$ height (pedestrian level), in which wind speed is a primary indicator of wind comfort in wind environment evaluation

(3) The line's length is as long as that of the calculation field, and the wind speed change in the entire computational domain was assessed

4.3.2. Deviation Calculation. The deviation is defined as

$$
\Delta=\frac{\int_{a}^{b}\left|f_{1} x-f_{2}(x)\right| \mathrm{d} x}{\int_{a}^{b} f_{1}(x) \mathrm{d} x},
$$

where $a$ and $b$ are the range of computational domain; $b-a=l$ is the length of the computational domain along the reference line; $f_{1}(x)$ and $f_{2}(x)$ are the fitting curves, respectively, for pre- and postconsolidation wind speed along the reference line.

Since the output value of CFD software is the wind speed at the reference line, it is difficult to form relevant fitting functions. To simplify this, this research inserted 1000 interpolation points into the initial data points according to the interpolation algorithm to get the following two sets of data points:

$$
\begin{aligned}
& f_{1}(x): y_{1}, y_{2}, \ldots, y_{1000} \\
& f_{2}(x): z_{1}, z_{2}, \ldots, z_{1000}
\end{aligned}
$$

Then, 
TABLe 2: Orthogonal experiment table L16 $\left(4^{5}\right)$.

\begin{tabular}{|c|c|c|c|c|c|}
\hline \multirow{2}{*}{ Test number } & \multicolumn{5}{|c|}{ Column number } \\
\hline & 1 & 2 & 3 & 4 & 5 \\
\hline 1 & I & $\mathrm{I}$ & $\mathrm{I}$ & $\mathrm{I}$ & $\mathrm{I}$ \\
\hline 2 & I & II & II & II & II \\
\hline 3 & I & III & III & III & III \\
\hline 4 & I & IV & IV & IV & IV \\
\hline 5 & II & I & II & III & IV \\
\hline 6 & II & II & I & IV & III \\
\hline 7 & II & III & IV & $\mathrm{I}$ & II \\
\hline 8 & II & IV & III & II & I \\
\hline 9 & III & I & III & IV & II \\
\hline 10 & III & II & IV & III & I \\
\hline 11 & III & III & $\mathrm{I}$ & II & IV \\
\hline 12 & III & IV & II & $\mathrm{I}$ & III \\
\hline 13 & IV & I & IV & II & III \\
\hline 14 & IV & II & III & I & IV \\
\hline 15 & IV & III & II & IV & I \\
\hline 16 & IV & IV & I & III & II \\
\hline
\end{tabular}

$$
\Delta=\frac{\left|y_{1}-z_{1}\right| * \Delta x+\left|y_{2}-z_{2}\right| * \Delta x+\cdots+\left|y_{m}-z_{m}\right| * \Delta x}{y_{1} * \Delta x+y_{2} * \Delta x+\cdots+y_{m} * \Delta x}=\frac{\sum_{i=1}^{m}\left|y_{i}-z_{i}\right|}{\sum_{i=1}^{m} y_{i}}
$$

4.4. CFD Simulation Settings. ANSYS Fluent 16.1 was used for CFD simulation and the simulation settings are shown in Table 3.

Considering a large number of simulation experiments required in the research, the standard $k-\varepsilon$ model was used for the advantage of low computing cost, little fluctuation, high accuracy in calculation, and extensive application in lowspeed turbulence. Computational domain and the settings of boundary condition and grid resolution referred to the CFD simulation standard of Architectural Institute of Japan (AIJ) [19]. To decrease the systematic error created by model simplification and coordination, the simplified model had the same grid expansion ratio, grid resolution, and boundary condition as the original model.

\section{Data Analysis}

5.1. Analysis of Single Factor Experiment. Figure 5 displays the changes of experiment deviation $\Delta$ in different levels in the four single factor tests (i.e., $B / L, W / L, D / L$, and $H / L$ ). Some findings are presented as follows.

Deviation $\triangle$ has a positive correlation with $B / L$ and $D / L$ (see Figures 5(a) and 5(c)), which indicates that the velocity difference in the reference line after simplification expands with the increase of $B / L$ and $D / L$. This is because the space formed by consolidating the two buildings becomes larger and larger with the increase of building distance B and offset distance $D$, thus further blocking the downdraft and transverse airflow in the building gaps to increase the change of wind speed.

Deviation $\triangle$ has an approximate negative correlation with $W / L$ (see Figure 5(b)), and the difference in velocity decreases as $W / L$ increases. The reason is that the width of buildings has less influence on the wind shadow area, and with the increase of $\mathrm{W}$, the relative change of volume of building group before and after consolidation decreases, thus reducing disturbance on air ventilation.

Deviation $\triangle$ has an overall positive correlation with $H / L$ (see Figure 5(d)) and the fluctuation of data point is due to the scale change of the wind shadow area. When $H / L=0$, there is no barrier in front of the reference line; consolidation has no effect on the results. With the increase of $H / L$, the wind shadow area that is directly proportional to the building height becomes larger and thus, the difference of velocity is more affected by $H / L$.

In summary, the results of all single factor experiments indicate that factor $D / L$ has a great effect on the wind speed deviation followed by $B / L, H / L$, and $W / L$. The exact order of the influence is further confirmed by the following orthogonal experiment. Moreover, the experiment results are basically consistent with the theoretical prediction, thus being able to be a reference for the orthogonal experiment.

5.2. Supplemental Design of Orthogonal Experiment. According to the results of the single factor experiment, the monotonous zone of each factor was selected as its value range in the orthogonal experiment as follows: $B / L: 0-2, W /$ $L: 0-2, H / L: 0-2$, and $D / L: 0-1$. The value ranges of all factors were set to four levels with equal intervals and the levels were selected randomly to avoid systematic errors caused by human factors. The levels of the factors in the orthogonal experiment are presented in Table 4.

All of the four factors and four levels mentioned above were substituted to Table 2, thus getting Table 5 including 16 tests. The fifth column in Table 5 was retained as an error column for the interaction test. $L$ in the orthogonal experiment was $30 \mathrm{~m}$, the same as that in the single factor experiment. The parameters of 16 orthogonal tests were converted according to the horizontal distribution of the 
TABLE 3: Simulation settings in ANSYS Fluent.

Grid generation

Computational domain

Boundary condition

Operation pressure Model

Near wall treatment Solving algorithms

\section{Unstructured grid}

Grid expansion ratio: 1.2

Grid resolution: $\leq 1 / 10$ of each edge of the building

The distance between the building outer boundary and the computational domain edge: ceiling $\geq 5 \mathrm{H}$, inflow boundary $\geq 5 \mathrm{H}$, cross-range $\geq 5 \mathrm{H}$, outflow boundary $\geq 15 \mathrm{H}$

$$
X * Y * Z: 2000 \mathrm{~m} * 1500 \mathrm{~m} * 600 \mathrm{~m}
$$

Inflow boundary and ceiling boundary: velocity-inlet $-v=U_{10} *(Z / 10)^{0.3}$

Outflow boundary: pressure-outlet-101325 Pa

Lateral boundary: symmetry

Ground and building surface: wall $101325 \mathrm{~Pa}$

Standard $k-\varepsilon$ model

Enhanced wall functions SIMPLEC

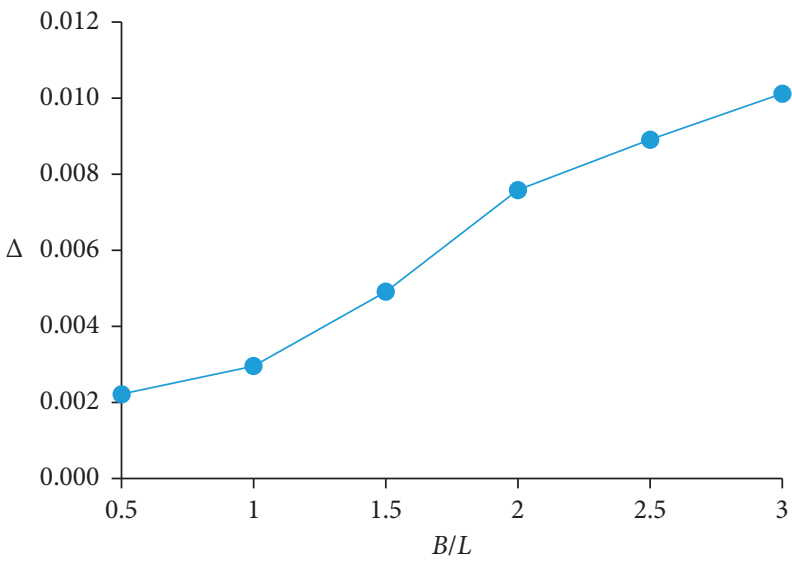

(a)

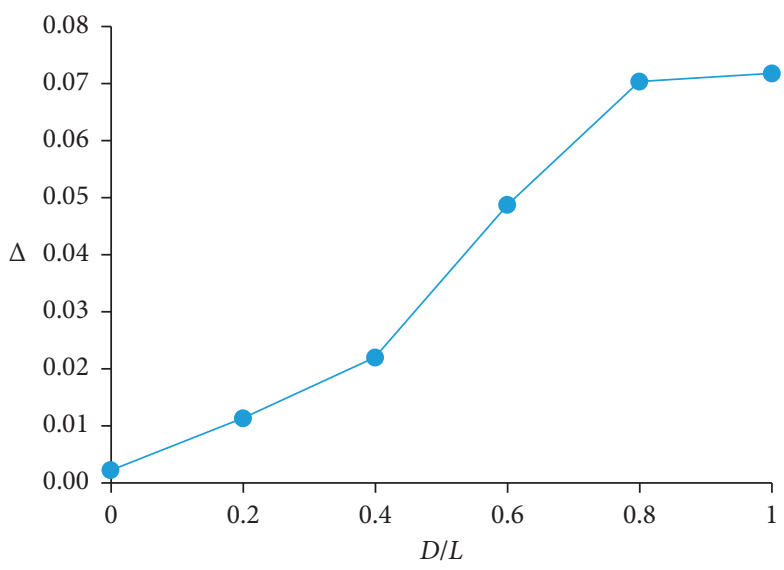

(c)

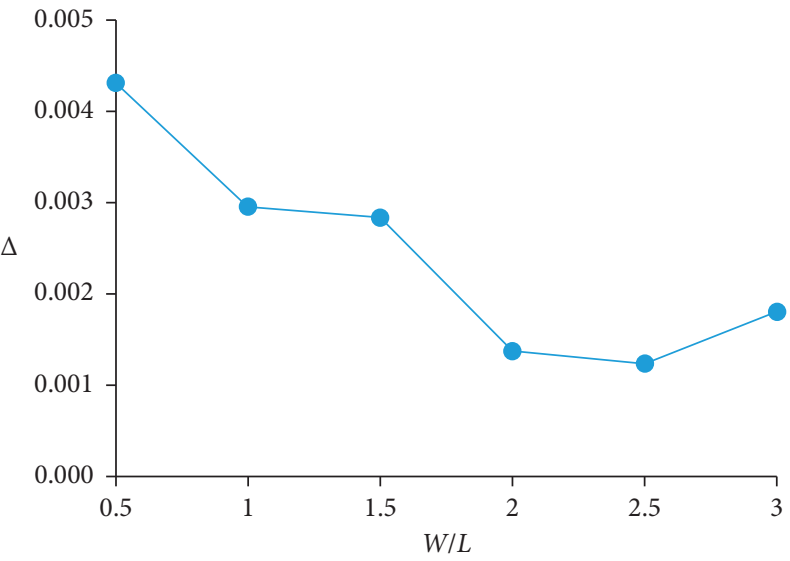

(b)

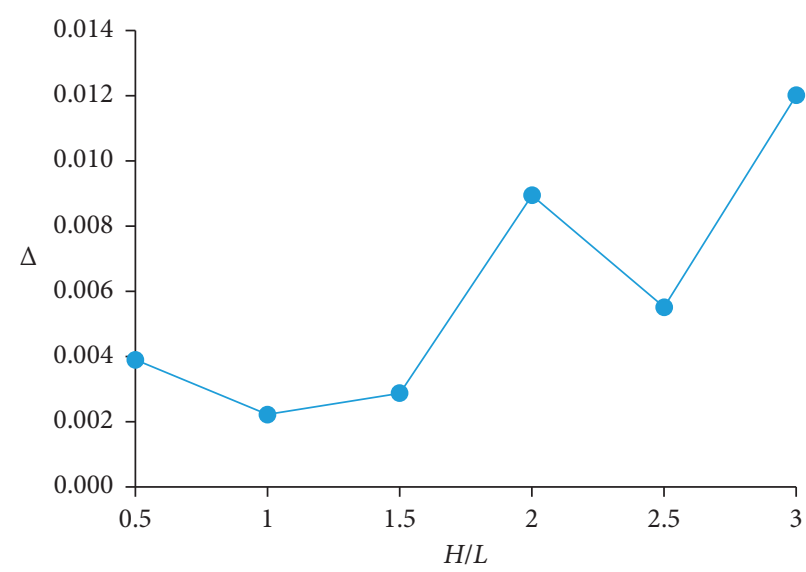

(d)

FIGURE 5: Changes of experiment deviation in different levels in four single factor tests. (a) Experiment deviation with $B / L$ changing from 0.5 to 3. (b) Experiment deviation with $W / L$ changing from 0.5 to 3. (c) Experiment deviation with $D / L$ changing from 0 to 1 . (d) Experiment deviation with $H / L$ changing from 0.5 to 3 .

TABLE 4: Level of the factors in the orthogonal experiment.

\begin{tabular}{lcccc}
\hline Level & (1) $(B / L)$ & (2) $(W / L)$ & (3) $(D / L)$ & (4) $(H / L)$ \\
\hline I & 1 & 0.5 & 1 & 1.5 \\
II & 0.5 & 1.5 & 0.5 & 2 \\
III & 2 & 1 & 0.25 & 0.5 \\
IV & 1.5 & 2 & 0.75 & 1 \\
\hline
\end{tabular}


TABLE 5: The orthogonal experiment design.

\begin{tabular}{|c|c|c|c|c|c|}
\hline Experiment number & (1) $(B / L)$ & (2) $(W / L)$ & (3) $(D / L)$ & (4) $(H / L)$ & (5) \\
\hline 1 & 1 & 0.5 & 1 & 1.5 & $\mathrm{I}$ \\
\hline 2 & 1 & 1.5 & 0.5 & 2 & II \\
\hline 3 & 1 & 1 & 0.25 & 0.5 & III \\
\hline 4 & 1 & 2 & 0.75 & 1 & IV \\
\hline 5 & 0.5 & 0.5 & 0.5 & 0.5 & IV \\
\hline 6 & 0.5 & 1.5 & 1 & 1 & III \\
\hline 7 & 0.5 & 1 & 0.75 & 1.5 & II \\
\hline 8 & 0.5 & 2 & 0.25 & 2 & I \\
\hline 9 & 2 & 0.5 & 0.25 & 1 & II \\
\hline 10 & 2 & 1.5 & 0.75 & 0.5 & $\mathrm{I}$ \\
\hline 11 & 2 & 1 & 1 & 2 & IV \\
\hline 12 & 2 & 2 & 0.5 & 1.5 & III \\
\hline 13 & 1.5 & 0.5 & 0.75 & 2 & III \\
\hline 14 & 1.5 & 1.5 & 0.25 & 1.5 & IV \\
\hline 15 & 1.5 & 1 & 0.5 & 1 & I \\
\hline 16 & 1.5 & 2 & 1 & 0.5 & II \\
\hline
\end{tabular}

orthogonal experiment table with the same CFD simulation settings.

5.3. Analysis of Orthogonal Experiment. Based on the CFD simulation, the deviations of the 16 orthogonal tests were obtained (see Table 6).

5.3.1. Intuitive Analysis. $K_{i}$ is the sum of the test results corresponding to level $i$ ( $i=\mathrm{I}$, II, III, and IV) in the same column of Table 5. According to the experiment results, $K$ values were obtained (see Table 7 ). The changes of $K$ values with the factor levels in the orthogonal experiment are shown in Figure 6.

The effect of each factor on the experiment result can be represented as

$$
\text { Range }(R)=\max \left\{K_{\mathrm{I}}, K_{\mathrm{II}}, K_{\mathrm{III}}, K_{\mathrm{IV}}\right\}-\min \left\{K_{\mathrm{I}}, K_{\mathrm{II}}, K_{\mathrm{III}}, K_{\mathrm{IV}}\right\} \text {. }
$$

Then, $R_{(3)}>R_{(1)}>R_{(4)}>R_{(2)}>R_{(5)}$ is found in Table 7. This indicates that $D / L$ has the most influence on the results, followed by $B / L$ and $H / L$, and $W / L$ is minimal; there is no interaction between different factors as well.

5.3.2. Variance Analysis. Furthermore, variance analysis was performed on the experiment results to accurately assess the effect of each factor. This is summarized in Table 8.

As shown in Table 8, under $\alpha=5 \%$ significance level, the effect of each factor on the experiment results is significant. For the error column, $F_{(3)}>F_{(1)}>F_{(4)}>F_{(2)}>F_{(5)}$; the greater the $F$ value is, the more significant its impact on the results is. It can be found that this result is consistent with that of intuitive analysis.

5.4. Suitability Verification. The suitability verification is to verify whether the results of the optimal scheme in the orthogonal experiment are consistent with the actual situations. According to the above results of the orthogonal experiment, the optimal values of four control parameters were determined as $B / L=1.5, W / L=2, H / L=0.5$, and $D /$ $L=0.25$, substituted into CFD simulation with the optimized deviation $\triangle=0.007687$, which is smaller than that of other 16 tests (see Table 6).

\section{Results and Discussion}

6.1. Determination of Control Parameters. Based on the above analysis, the optimal and suboptimal values of the four control parameters are presented in Table 9. Furthermore, by considering the significance of each factor $(D / L>B / L>H /$ $L>W / L)$, the value ranges of control parameters were determined as follows.

$B / L$ : according to the single factor experiment, $B / L$ was positively correlated with $\triangle$, the optimal value $B / L=1.5$. However, building groups should already be adjusted when $B / L=0$; thus, the value range of $B / L$ is $0-1.5$.

$W / L$ : according to the single factor experiment, $W / L$ was positively correlated with $\triangle$, the optimal value $W / L=2$. The effect of $W / L$ on the experiment results was not significant and the value of $W / L$ in actual building groups may be small; thus, the value range of $W / L$ can be extended to $0-2$.

$D / L$ : according to the single factor experiment, $D / L$ was positively correlated with $\triangle$, the optimal value $D / L=0.25$, and with the most significant effect on the experiments. Therefore, the optimal value was set as the upper limit of $D /$ $L$; its value range is $0-0.25$.

$H / L$ : according to the single factor experiment, $H / L$ was positively correlated with $\triangle$, the optimal value $H / L=0.5$. The effect of $H / L$ on the experiment results was not too significant; thus, the suboptimal value was selected as the upper limit of $H / L$, and its value range is $0-1$.

Although the simplified method proposed in this research is based on two building groups, its application can be extended to multiple-building groups when their relationships satisfy the constraints of the control indexes. Besides, the control indexes should be adjusted appropriately according to the actual scale of building groups and the demand accuracy of CFD simulation. 
TABLE 6: Deviations of the orthogonal experiment.

\begin{tabular}{lcccccccc}
\hline Experiment number & 1 & 2 & 3 & 4 & 5 & 6 & 7 & 8 \\
Deviation $\triangle$ & 0.067892 & 0.038909 & 0.021115 & 0.047969 & 0.026526 & 0.059733 & 0.05444 & 0.007824 \\
\hline Experiment number & 9 & 10 & 11 & 12 & 13 & 14 & 15 & 16 \\
Deviation $\triangle$ & 0.017716 & 0.021564 & 0.050074 & 0.014583 & 0.03658 & 0.011816 & 0.020159 & 0.016482 \\
\hline
\end{tabular}

TABLe 7: $K$ value of the orthogonal experiment.

\begin{tabular}{lcccrr}
\hline$K_{i}$ & $(1)(B / L)$ & (2) $(W / L)$ & (3) $(D / L)$ & (4) $(H / L)$ & 0.148731 \\
\hline$K_{\mathrm{I}}$ & 0.175884509 & 0.148714 & 0.1941814 & 0.133387 & 0.117439 \\
$K_{\mathrm{II}}$ & 0.148523534 & 0.132021 & 0.100177 & 0.085687 & 0.127546 \\
$K_{\mathrm{III}}$ & 0.103936222 & 0.145787 & 0.0584707 & 0.145576 & 0.136385 \\
$K_{\mathrm{IV}}$ & 0.085036869 & 0.086859 & 0.160552 & 0.063045 & 0.018945 \\
Range $(R)$ & 0.09084764 & 0.061856 & 0.1357107 & 0.075 \\
\hline
\end{tabular}

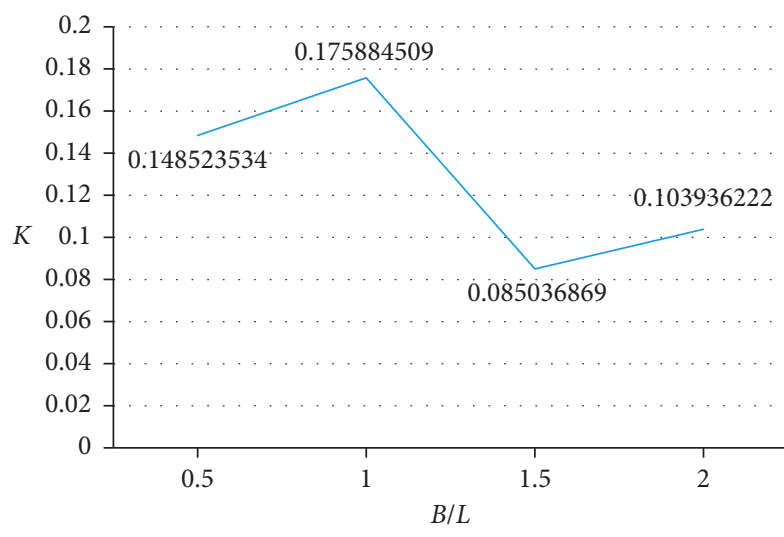

(a)

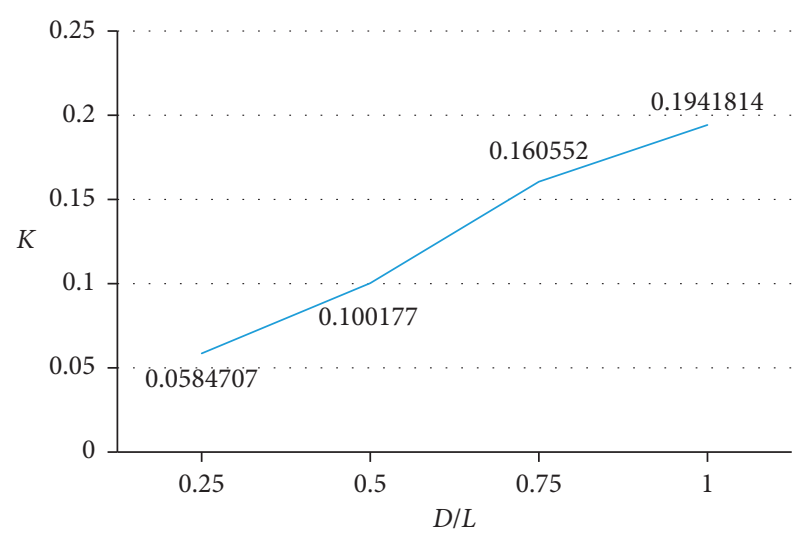

(c)

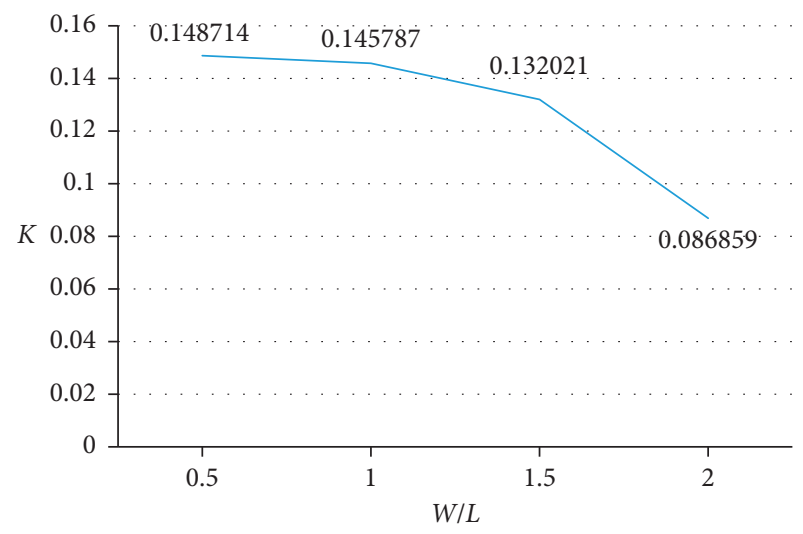

(b)

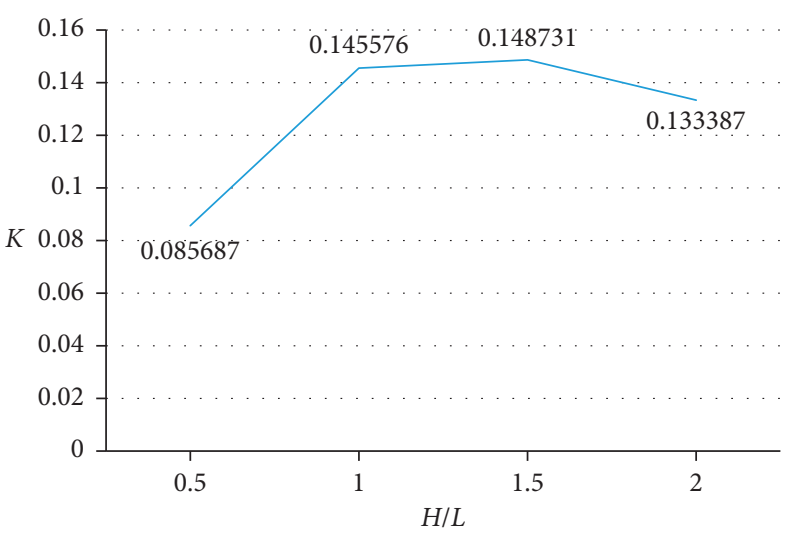

(d)

Figure 6: Changes of $K$ value with the factor levels in the orthogonal experiment. (a) Changes of $K$ value with different $B / L$ levels. (b) Changes of $K$ value with different $W / L$ levels. (c) Changes of $K$ value with different $D / L$ levels. (d) Changes of $K$ value with different $H / L$ levels.

TABLE 8: Variance analysis of the orthogonal experiment.

\begin{tabular}{lccccc}
\hline Column no. & SS & $\mathrm{d} f$ & MS & $F$ & Significance $(\alpha=0.05)$ \\
\hline (1) & 0.00128464 & 3 & 0.0004282 & 25.99792 & Outstanding \\
(2) & 0.000613442 & 3 & 0.0002045 & 12.41453 & Outstanding \\
(3) & 0.002761893 & 3 & 0.0009206 & 55.89385 & Outstanding \\
(4) & 0.000639416 & 3 & 0.0002131 & 12.94018 & Outstanding \\
(5) & $4.94132 E-05$ & 3 & $1.647 E-05$ & 1 & \\
\hline
\end{tabular}

Note: $S S_{j}$ is the sum of the square of deviations on the same column $j(j=$ (1), (2), (3), (4), and (5) in the orthogonal table, representing the difference of experiment results; $\mathrm{d} f_{j}$ represents the degree of freedom, equal to the number of factor levels with minus $1 ; \mathrm{MS}_{j}$ is the average value of $\mathrm{SS}_{j}$, equal to $\mathrm{SS}_{j} / \mathrm{d}_{j} ; F$ equal to $\mathrm{MS}_{j} / \mathrm{MS}_{5}$ is to evaluate the significance of each factor. 
TABLE 9: Optimal and suboptimal value of the control parameters.

\begin{tabular}{lcccc}
\hline & $B / L$ & $W / L$ & $D / L$ & $H / L$ \\
\hline Optimal value & 1.5 & 2 & 0.25 & 0.5 \\
Suboptimal value & 2 & 1.5 & 0.5 & 1 \\
\hline
\end{tabular}

6.2. Discussion. According to data analysis, the changing trend of $B / L$ in the orthogonal experiment was different from that in the single factor experiment; this may be caused by the interaction between $B / L$ and other factors. Although, as an independent factor, the effect of $B / L$ on deviation was clear, the relationship might change after introducing the other factors. In the orthogonal experiment, the ratios of $B$, $W, H$, and $D$ to $L$ were selected as the factors which are not completely independent of each other. However, this kind of interaction can be verified by introducing other types of parameters in future experiments to figure out more reasonable and accurate control parameters.

\section{Case Study}

To verify the proposed simplified method, the model of a building group containing a total of 620 buildings with a height from $3 \mathrm{~m}$ to $78 \mathrm{~m}$ at Tsinghua University in Beijing was built (see Figure 7), with the size of $1,855 \mathrm{~m}$ along the east-west direction and 2,255 $\mathrm{m}$ along the south-north direction. The buildings whose control parameters met the requirements in Table 9 with identical height and regular arrangement were selected to test the validity of the control indexes. The appropriate building models were first simplified (consolidated); then, the simulation of the wind environment was conducted.

7.1. Model Simplification. To simplify the building group model, we considered the following two principles:

(1) The simplified objects were rectangular building groups, in which the buildings had similar shapes and their three-dimensional variances were less than 3. Especially, the buildings with less than $10 \mathrm{~m}$ height had a few effects on the overall wind field and thus could be consolidated directly. However, considering the effect of the uniqueness of buildings on the wind field, special-shaped or super high-rise buildings were not simplified in this research.

(2) The spatial planning of land use and the function of buildings should be considered when they were simplified. The buildings in different use of land could not be simplified, such as roads, parks, and rivers.

According to these principles, the simplification process of the building group model using the simplified method and the control parameters is shown in Figure 8.

As shown in Figure 9, the simplified building models cover many areas decreasing the number of buildings from 620 to 395 (by $36.3 \%$ ).

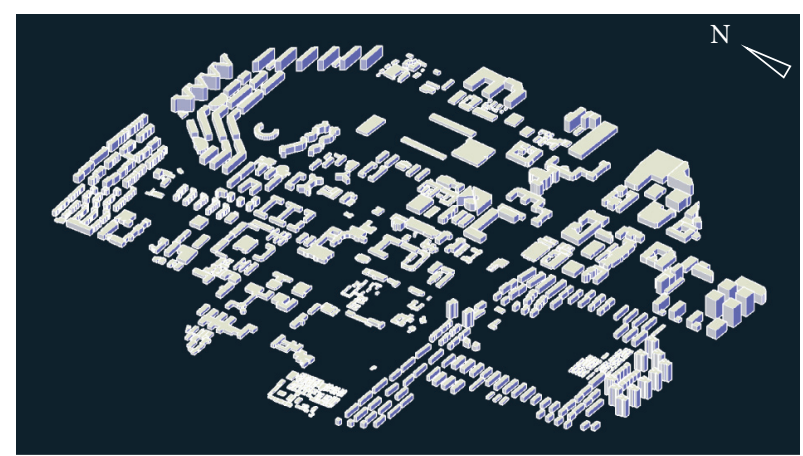

FIGURE 7: The original 3D model of the building group.

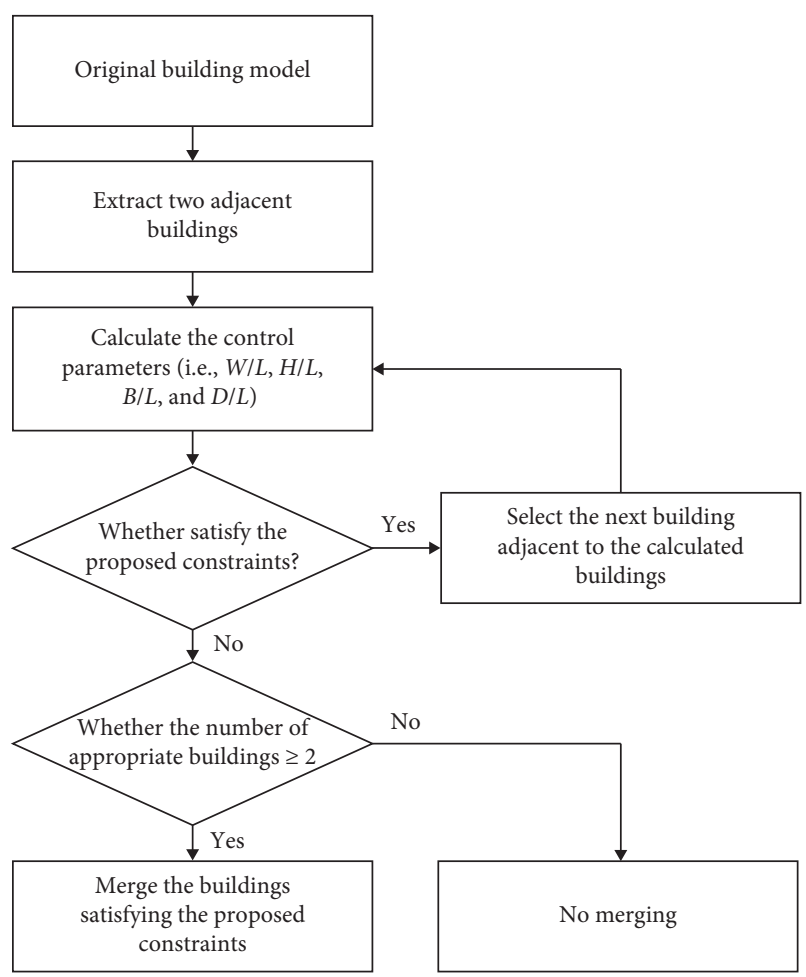

FIGURE 8: The simplification process of the building group model.

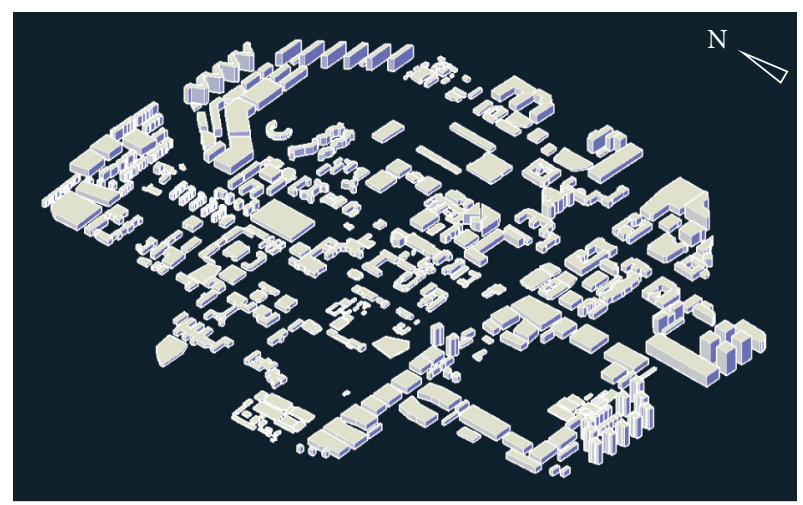

Figure 9: The simplified 3D model of the building group. 
TABLE 10: The settings of various simulations in ANSYS Fluent.

\begin{tabular}{|c|c|}
\hline Items & Contents \\
\hline Simulation software & ANSYS fluent 16.1 \\
\hline Grid generation & $\begin{array}{l}\text { Unstructured grid: the building grid size is } 4 \mathrm{~m} \text {, the ground grid size is } 10 \mathrm{~m} \text {, and the global grid size is } 100 \mathrm{~m} \text {; the } \\
\text { size of the simplified building grid becomes } 8 \mathrm{~m} \text {, while other dimensions remain unchanged }\end{array}$ \\
\hline $\begin{array}{l}\text { Computational } \\
\text { domain }\end{array}$ & $\begin{array}{l}\text { The distances between the building outer boundary and the computational domain edge: ceiling }=5 H \text {, inflow } \\
\text { boundary }=5 H \text {, cross-range }=5 H \text {, and outflow boundary }=15 H ; X * Y * Z: 2635 \mathrm{~m} * 3815 \mathrm{~m} * 468 \mathrm{~m} \\
\text { Inflow boundary and ceiling boundary: velocity-inlet- }-\mathrm{v}=v 0 *(Z / 10)^{0.3}\end{array}$ \\
\hline Boundary condition & $\begin{array}{c}\text { Outflow boundary: pressure-outlet-_ } 101325 \mathrm{~Pa} \\
\text { Lateral boundary: symmetry } \\
\text { Ground and building surface: wall }\end{array}$ \\
\hline Pressure & $101325 \mathrm{~Pa}$ \\
\hline Model & Standard $k-\varepsilon$ model \\
\hline Near wall treatment & Standard wall treatment \\
\hline Solving algorithms & SIMPLEC \\
\hline Convergence criteria & The speed of the monitoring point reaches a steady value \\
\hline
\end{tabular}

\subsection{CFD Simulation}

7.2.1. Settings of CFD Simulation. Considering the characteristics of local climate, model size and complexity, computing capability, etc., the settings of CFD simulation modeling are presented in Table 10.

7.2.2. Comparison of Grids. The result of the CFD simulation indicates that the quantity of the grids in the flow field can be reduced by the proposed simplified method with improved quality. As shown in Figure 10, considering the complicated geometric configuration of the building group model, the Octree algorithm in ANSYS ICEM CFD instead of structured grids was applied to generate the tetrahedral unstructured grids whose size and quantity were subject to computing resources. Compared with the original grid size in Table 10, the size of the simplified building grid became $8 \mathrm{~m}$ with other dimensions unchanged to reduce the impact of different overall grid resolutions on the calculation results. In the end, the number of tetrahedral grids was reduced from $8,832,199$ to $7,766,778$ (by $12.1 \%$ ).

The comparison of grid quality before and after simplification is shown in Figure 11. The closer the grid quality value is to 1 , the better the grid quality is. It is obvious that the overall grid quality is improved after simplification. This is because the reduction of the narrow space in the simplified model can smooth the tetrahedral grid transition under a given size and reduce grid problems such as repeating units and unconnected vertices.

7.2.3. Simulation Results. Airflow direction, speed, and relevant changes in urban areas are the main factors affecting the simulation of the wind environment of building groups on a large scale. To satisfy the requirement of grid accuracy, a $10 \mathrm{~m}$ high plane above the ground was selected as the reference surface to analyze the flow field. The velocity contour cloud maps and velocity vector maps in the reference surface before and after simplification are shown in Figures 12 and 13. The following findings were achieved:

(1) Compared with the original model, there were few changes of cloud map in the open space and the street

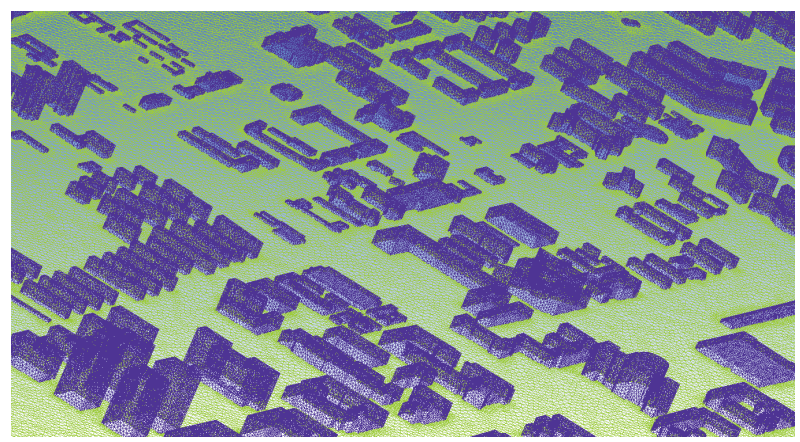

Figure 10: The grids of the original model.

valley of the simplified model, except for unstable wind speed in the simplified area. Moreover, the distribution of wind speed in most areas (e.g., areas (1), (2), and (3) marked in Figure 12) after simplification was almost the same as that before simplification.

(2) Merging adjacent buildings not only blocked original air passages but also caused a few changes in wind direction around the simplified building models. However, the overall distribution of wind direction in the flow field (e.g., areas (4), (5), and (6) marked in Figure 13) after simplification was also almost the same as that before simplification.

(3) Although the comparison of the wind fields before and after simplification indicated that the simplification of the building group model had an unavoidable impact on the local wind environment, the simulation results were acceptable at the urban level because there were no significant airflow changes in the main ventilation paths.

7.2.4. Summary. Compared with the original simulation, the quantity of the simplified grids was reduced by $12.1 \%$. The simulation results indicated that the proposed simplified method with the exact control parameters was feasible for wind environment simulation on the urban scale but saved computational resources and improved computational efficiency. Besides, the improvement of computational 


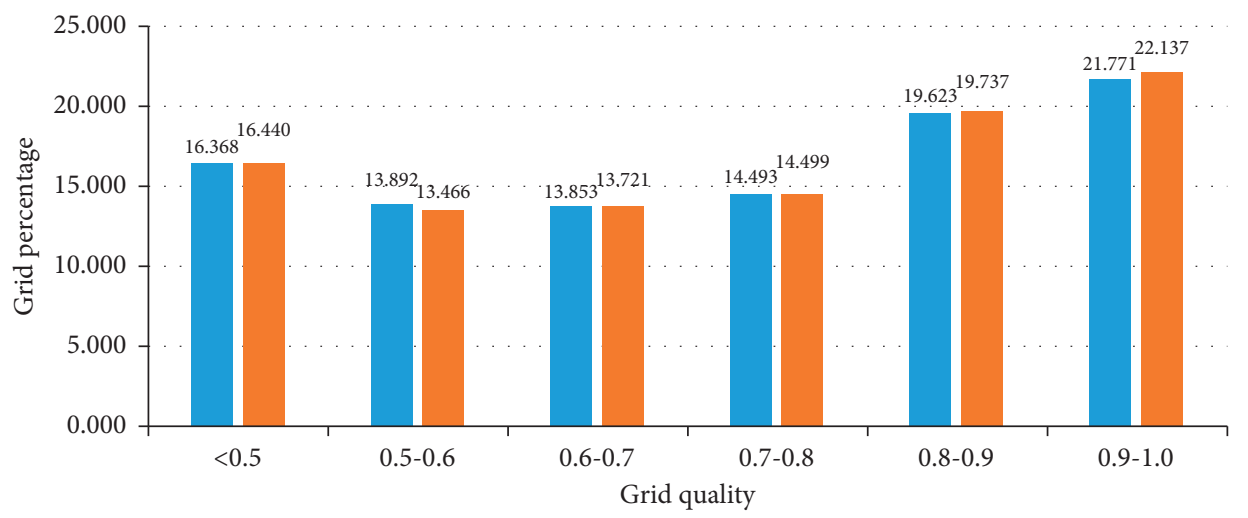

- Before simplification

- After simplification

Figure 11: Comparison of grid quantity before and after simplification.

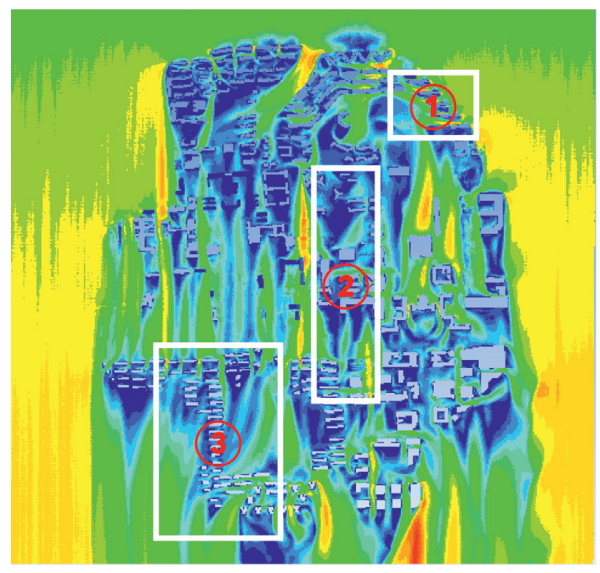

(a)

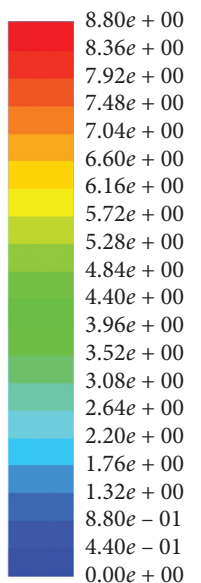

$0.00 e+00$
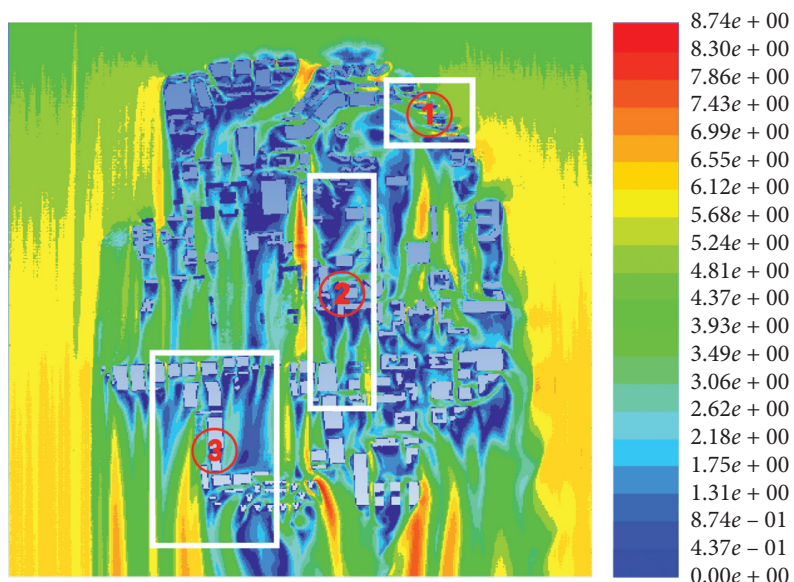

(b)

FIGURE 12: Velocity contour cloud maps before and after simplification. (a) Velocity contour cloud map before simplification. (b) Velocity contour cloud map after simplification.

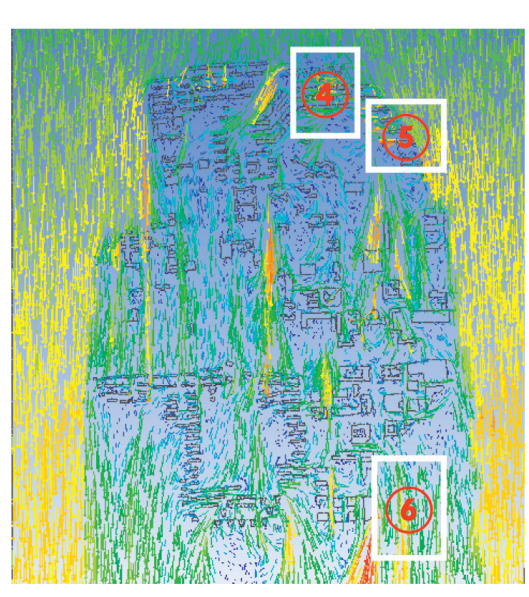

(a)

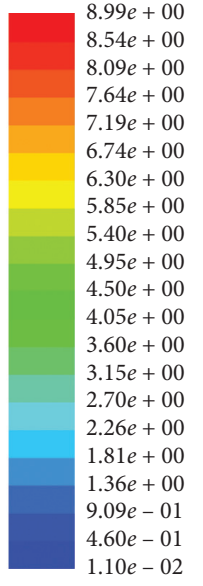

$1.60 e-01$

FIGURE 13: Velocity vector maps before and after simplification. (a) Velocity vector map before simplification. (b) Velocity vector map after simplification. 
efficiency in this research was subject to the strict selection criteria and simplification process for building group models. In the future practical applications, especially for the CFD simulation of building groups in an urban scale (size between $10 \mathrm{~km}^{2}$ and $20 \mathrm{~km}^{2}$ ) with denser areas (such as residential areas), higher grid accuracy, and more grids, the simplified method will be much more valuable and efficient.

\section{Conclusion}

A simplified method for the model of building groups in CFD simulation is proposed in this article and the appropriate value ranges of the four control parameters are determined as $B / L \in\{0,1.5\}, W / L \in\{0,2\}, D / L \in\{0,0.25\}$, and $H / L \in\{0,1\}$ using single factor experiment and orthogonal experiment. According to the variance analysis on the results of the orthogonal experiment, $D / L$ has the most effect on deviation $\triangle$, followed by $B / L, H / L$, and $W / L$. When the geometric relationship of a couple of buildings satisfies the control indexes, the simplified method is applicable. In order to verify the proposed method, the simulation model of the building group in Tsinghua University is built and analyzed in the case study. The results indicate that the proposed method can effectively reduce the number of grids generated in CFD simulation and improve the efficiency of simulation without decreasing the performance of simulation, especially in the simulation of a large scale of building groups. Therefore, it will extend the application of CFD simulation in the urban-level wind environment analysis.

However, there are also some limitations to this research. For example, the problems such as system errors, strong dissipativity, and low computational accuracy can be caused by the standard $k-\varepsilon$ turbulence model. It can be substituted with the LES model or tunnel test data for higher accuracy results in future research. Besides, as the selected factors are not completely independent of each other, there are some interactions between these factors. This can be verified by introducing other types of parameters such as topography, inflow angle, and building group layout in the future.

\section{Data Availability}

The data used in this manuscript will be provided upon request.

\section{Disclosure}

This article is an extension of the conference paper " $A$ Simplified Method for the Model of Building Groups in CFD Simulation" published in the International Conference on Construction and Real Estate Management (ICCREM) 2017.

\section{Conflicts of Interest}

The authors declare that they have no conflicts of interest.

\section{Acknowledgments}

This work was supported by the NSFC-Zhejiang Joint Fund for the Integration of Industrialization and Informatization
(Grant no. U1709212) and the Tsinghua University-Glodon Joint Research Centre for Building Information Model (RCBIM). The authors also thank Prof. Xinzheng $\mathrm{Lu}$ at Tsinghua University for providing the building data in the case study.

\section{References}

[1] C. Yuan, L. Norford, R. Britter, and E. Ng, "A modellingmapping approach for fine-scale assessment of pedestrianlevel wind in high-density cities," Building and Environment, vol. 97, no. 15, pp. 152-165, 2016.

[2] B. Wang, L. D. Cot, L. Adolphe, S. Geoffroy, and J. Morchain, "Estimation of wind energy over roof of two perpendicular buildings," Energy and Buildings, vol. 88, no. 1, pp. 57-67, 2015.

[3] Y. Huang, X. Hu, and N. Zeng, "Impact of wedge-shaped roofs on airflow and pollutant dispersion inside urban street canyons," Building and Environment, vol. 44, no. 12, pp. 2335-2347, 2009.

[4] G. M. Stavrakakis, E. Tzanaki, V. I. Genetzaki, G. Anagnostakis, G. Galetakis, and E. Grigorakis, "A computational methodology for effective bioclimatic-design applications in the urban environment," Sustainable Cities and Society, vol. 4, pp. 41-57, 2012.

[5] J. D. Anderson, Computational Fluid Dynamics: The Basics with Applications, McGraw-Hill, New York, NY, USA, 1995.

[6] P.-C. Liu, H.-T. Lin, and J.-H. Chou, "Evaluation of buoyancy-driven ventilation in atrium buildings using computational fluid dynamics and reduced-scale air model," Building and Environment, vol. 44, no. 9, pp. 1970-1979, 2009.

[7] T. Kobayashi, T. Chikamoto, and K. Osada, "Evaluation of ventilation performance of monitor roof in residential area based on simplified estimation and CFD analysis," Building and Environment, vol. 63, pp. 20-30, 2013.

[8] J. I. Perén, T. van Hooff, B. C. C. Leite, and B. Blocken, "Impact of eaves on cross-ventilation of a generic isolated leeward sawtooth roof building: windward eaves, leeward eaves and eaves inclination," Building and Environment, vol. 92, pp. 578-590, 2015.

[9] R. Teppner, B. Langensteiner, W. Meile, G. Brenn, and S. Kerschbaumer, "Air change rates driven by the flow around and through a building storey with fully open or tilted windows: an experimental and numerical study," Energy and Buildings, vol. 80, pp. 570-583, 2014.

[10] C. M. Mak, J. L. Niu, C. T. Lee, and K. F. Chan, "A numerical simulation of wing walls using computational fluid dynamics," Energy and Buildings, vol. 39, no. 9, pp. 995-1002, 2007.

[11] B. Blocken, J. Carmeliet, and T. Stathopoulos, "CFD evaluation of wind speed conditions in passages between parallel buildings-effect of wall-function roughness modifications for the atmospheric boundary layer flow," Journal of Wind Engineering and Industrial Aerodynamics, vol. 95, no. 9-11, pp. 941-962, 2007.

[12] T. Ayata and O. Y1ldız, "Investigating the potential use of natural ventilation in new building designs in Turkey," Energy and Buildings, vol. 38, no. 8, pp. 959-963, 2006.

[13] O. S. Asfour, "Prediction of wind environment in different grouping patterns of housing blocks," Energy and Buildings, vol. 42, no. 11, pp. 2061-2069, 2010.

[14] J. O. P. Cheung and C.-H. Liu, "CFD simulations of natural ventilation behaviour in high-rise buildings in regular and staggered arrangements at various spacings," Energy and Buildings, vol. 43, no. 5, pp. 1149-1158, 2011. 
[15] A. Cao, Q. Li, and Q. Meng, "Effects of orientation of urban roads on the local thermal environment in Guangzhou city," Procedia Engineering, vol. 121, pp. 2075-2082, 2015.

[16] R. Ramponi, B. Blocken, L. B. de Coo, and W. D. Janssen, "CFD simulation of outdoor ventilation of generic urban configurations with different urban densities and equal and unequal street widths," Building and Environment, vol. 92, pp. 152-166, 2015.

[17] J. Hang, Z. Luo, M. Sandberg, and J. Gong, "Natural ventilation assessment in typical open and semi-open urban environments under various wind directions," Building and Environment, vol. 70, no. 15, pp. 318-333, 2013.

[18] C. Yuan, E. Ng, and L. K. Norford, "Improving air quality in high-density cities by understanding the relationship between air pollutant dispersion and urban morphologies," Building and Environment, vol. 71, no. 1, pp. 245-258, 2014.

[19] Y. Tominaga, A. Mochida, R. Yoshie et al., "AIJ guidelines for practical applications of CFD to pedestrian wind environment around buildings," Journal of Wind Engineering and Industrial Aerodynamics, vol. 96, no. 10-11, pp. 1749-1761, 2008.

[20] Z. Wu, T. Yan, and X. Fu, CFD Simulation Technology Based Analysis on Urban Wind Environment of Shenzhen, The 3rd China Construction Quality Forum, Shenzhen, China, 2009.

[21] L. Yang, Green Building Design: Wind Environment of Building, Tongji University Press, Shanghai, China, 2014.

[22] X. Y. Chen, B. Zheng, K. M. Hou, and D. K. Zhong, Building Design and Natural Ventilation, China Electric Power Press, Beijing, China, 2012.

[23] J. Franke, A. Hellsten, H. Schlünzen, and B. Carissimo, "The COST 732 best practice guideline for CFD simulation of flows in the urban environment: a summary," International Journal of Environment and Pollution, vol. 44, no. 1-4, pp. 419-427, 2011. 